\title{
Long non-coding RNA MEG3 functions as a tumour suppressor and has prognostic predictive value in human pancreatic cancer
}

\author{
LING MA $^{1,2}$, FENG WANG $^{1}$, CHONG DU $^{1}$, ZHENGKUI ZHANG $^{1}$, HUAHU GUO $^{1}$, \\ XUEHAI XIE ${ }^{1}$, HONGQIAO GAO ${ }^{1}$, YAN ZHUANG ${ }^{1}$, MARKO KORNMANN ${ }^{3}$, \\ HONG GAO $^{2}$, XIAODONG TIAN ${ }^{1}$ and YINMO YANG ${ }^{1}$ \\ ${ }^{1}$ Department of General Surgery, Peking University First Hospital, Beijing 100034; ${ }^{2}$ Department of Surgical Oncology, \\ Peking University Ninth School of Clinical Medicine (Beijing Shijitan Hospital, Capital Medical University), \\ Beijing 100038, P.R. China; ${ }^{3}$ Clinic of General, Visceral and Transplantation Surgery, \\ University of Ulm, D-89081 Ulm, Germany
}

Received August 9, 2017; Accepted December 29, 2017

DOI: $10.3892 /$ or.2018.6178

\begin{abstract}
Long non-coding RNA (lncRNA) MEG3 has been demonstrated to be a tumour suppressor in many malignancies. However, the functional role of MEG3 in pancreatic cancer (PC) is unclear. In this study, the expression pattern of MEG3 was evaluated in 25 samples of microdissected PC tissues and 8 PC cell lines and was compared to the expression in adjacent non-cancerous tissues and a human pancreatic normal epithelial cell line. Loss of MEG3 expression was observed in both the cancerous tissues and cancer cell lines. Although the absence of expression of MEG3 was not statistically correlated to either histological grade or TNM stage in the 25 cases, the prognosis was significantly worse. MEG3 knockdown enhanced cell proliferation, promoted cell migration and invasion, induced epithelial-mesenchymal transition (EMT), increased the sphere-forming ability and cancer stem cell (CSC) properties, and decreased the chemosensitivity to gemcitabine in vitro. In contrast, forced expression of MEG3 resulted in a reverse effect. In conclusion, MEG3 functions as a tumour suppressor in human PC. The underlying cause of the poor prognosis induced by low levels of MEG3 expression in PC patients might involve EMT induction, enhanced CSC phenotypes and reduced chemoresistance, all of which might be associated with Snail activation.
\end{abstract}

\section{Introduction}

Pancreatic cancer (PC) is one of the most aggressive solid tumours, and ranks as the fourth leading cause of cancer-related

Correspondence to: Professors Xiaodong Tian or Yinmo Yang, Department of General Surgery, Peking University First Hospital, 8th Xishiku Street, Beijing 100034, P.R. China

E-mail: tianxiaodong@pkufh.cn

E-mail: yangyinmo@263.net

Key words: MEG3, pancreatic cancer, cell proliferation, epithelialmesenchymal transition, cancer stem cells, chemosensitivity death with a 5-year survival rate of only $7 \%$ in the United States (1). In 2017, PC is likely to overtake breast carcinoma as the third leading cause of cancer-related death in European countries (2). Due to its ample ability to invade surrounding tissue and early metastasis to distal organs, as well as the natural resistance to chemoradiotherapy, PC is associated with a poor patient prognosis. Therefore, it is urgent to elucidate the biological mechanisms responsible for the tumourigenesis of human PC and explore novel potential diagnostic biomarkers and therapeutic targets.

Long non-coding RNAs (lncRNAs) are a class of non-coding RNAs (ncRNAs) with a length of more than $200 \mathrm{nt}$. Instead of coding for proteins, lncRNAs function as regulators of epigenetic modifications, transcriptional and post-transcriptional regulation. IncRNAs have been found to play vital roles in not only physiological processes, but also tumour progression.

Maternally expressed gene 3 (MEG3) is a maternally expressed imprinted gene approximately $1.6 \mathrm{~kb}$ nucleotides in length (GenBank NR_002766) that is located on human chromosome 14q32.3. It was first identified as a human homologue to mouse maternal imprinting gene Glt2 (gene-trap locus 2) in 2000 (3). Without obvious open reading frames in its sequence, MEG3 functions as a regulatory ncRNA instead of coding for proteins. Conserved expression of MEG3 has been detected in many human tissues and organs, especially in the brain and pituitary gland (4). Meanwhile, abnormal downregulation of MEG3 was reported in malignant meningioma (5), pituitary tumours (4), lung adenocarcinoma (6), colorectal carcinoma $(7,8)$, hepatocellular cancer (9), urothelial carcinoma (10), osteosarcoma (11) and adult T-cell leukaemia (12). Furthermore, MEG3 was recognized as a tumour suppressor, and the significant absence of expression of MEG3 caused by gene deletion, promoter hypermethylation or hypermethylation of the intergenic differentially methylated region could contribute to tumourigenesis. Additionally, loss of MEG3 expression has been verified to primarily promote cell proliferation in multiple cancers via both p53-dependent and p53-independent pathways $(4,13)$. Zhao et al discovered that cyclic AMP (cAMP) could induce MEG3 expression, 
which in turn activated both the p53 and Rb pathways to modulate cell proliferation (14). Moreover, Liu et al found that MEG3 overexpression in lung adenocarcinoma cells increased their chemosensitivity to cisplatin by inhibiting cell proliferation and inducing apoptosis (6). On the other hand, Gordon et al reported that downregulation of MEG3 could inhibit angiogenesis via VEGF and Notch signaling to repress tumour metastasis (15). It has been confirmed that MEG3 is associated with survival in many types of carcinoma. Tian et al revealed that patients with low MEG3 expression in osteosarcoma had a shorter overall survival (11); similar conclusions were drawn regarding colorectal cancer (8) and retinoblastoma (16). Chang et al successfully delivered MEG3 RNA into hepatic cancer cells using a designed vector based on MS2 virus-like particles (VLPs) crosslinked with GE11 polypeptide and observed that cell proliferation, colony formation and cell invasion were all significantly suppressed (17).

To date, the relationship between MEG3 and human PC has not been fully elucidated. The aim of this study was to reveal whether MEG3 contributes to PC progression and to identify the underlying regulatory mechanisms. MEG3 expression was evaluated in both PC tissues and cell lines. The correlation of MEG3 expression in PC tissues with various clinicopathological characteristics and overall survival were also analysed. PC cells were transfected with either siRNAs or lentiviral vectors to change the expression of MEG3 in order to investigate the biological function of MEG3 on cell proliferation, cell migration and invasion, EMT, CSC properties and chemoresistance in vitro.

\section{Materials and methods}

Human tissue samples. Tissue samples from 25 PC patients who underwent surgical resection of their tumours were obtained from Peking University First Hospital. Both tumours and adjacent non-cancerous tissues were frozen in liquid nitrogen and stored at $-80^{\circ} \mathrm{C}$ until further use. This study was approved by the Ethics Committee of Peking University First Hospital, and written informed consent was provided by each patient.

Laser captured microdissection. Given the tissue heterogeneity of PC, it was necessary to isolate pure malignant cells from heterogeneous tissue sections via laser capture microdissection (LCM) before further examination. The tissue samples were processed into frozen sections using PEN glass microscope slides specifically designed for LCM. After the target cells were stained with haematoxylin and eosin $(\mathrm{H} \& \mathrm{E})$, they were recognized and labelled by computer. Then, a Leica LMD7000 instrument (Leica, Wetzlar, Germany) was used to separate the malignant pancreatic ducts from the frozen sections.

Cell culture. The human PC cell lines SW 1990, COLO357, MIA PaCa-2, T3M4, AsPC-1, BxPC-3, CAPAN-1 and PANC-1, as well as human pancreatic normal epithelial cell line hTERT-HPNE, were maintained in our laboratory. MIA PaCa-2, COLO357 and PANC-1 cells were cultured in Dulbecco's modified Eagle's medium (DMEM, Gibco,
Waltham, MA, USA), and T3M4, AsPC-1, BxPC-3 and CAPAN-1 cells were cultured in RPMI-1640 medium (Gibco) at $37^{\circ} \mathrm{C}$ in a humidified atmosphere containing $5 \% \mathrm{CO}_{2}$. Both types of media were supplemented with $10 \%$ fetal bovine serum (FBS, Gibco), $100 \mathrm{U} / \mathrm{ml}$ penicillin and $100 \mu \mathrm{g} / \mathrm{ml}$ streptomycin. SW 1990 cells were cultured in Leibovitz's L-15 medium supplemented with $10 \%$ FBS and maintained at $100 \%$ atmospheric air. hTERT-HPNE cells was cultured in $75 \%$ DMEM without glucose and 25\% Medium M3 Base (InCell Corp., San Antonio, TX, USA) supplemented with 5\% FBS, $5.5 \mathrm{mM}$ D-glucose $(1 \mathrm{~g} / \mathrm{l}), 10 \mathrm{ng} / \mathrm{ml}$ human recombinant EGF and $750 \mathrm{ng} / \mathrm{ml}$ puromycin.

RNA extraction and real-time quantitative PCR. Total RNA was extracted from microdissected PC tissues using an RNeasy Micro kit (Qiagen, Hilden, Germany) and from PC cell lines using TRIzol reagent (Invitrogen, Carlsbad, CA, USA) according to the respective manufacturers' protocols. The levels of MEG3, E-cadherin, N-cadherin, Snail, Vimentin, Nanog and Oct4 expression were measured using real-time quantitative PCR (qPCR). Eukaryotic 18S rRNA (18S) was adopted as the endogenous control for the human tissues, and glyceraldehyde-3-phosphate dehydrogenase (GAPDH) was used for the cells. MEG3 primers were purchased from RiboBio (Guangzhou, China). The primers for the other detected genes were as follows: 5'-GCCTCCTGAAAAGAG AGTGGAAG-3' (sense) and 5'-TGGCAGTGTCTCTCCAAA TCCG-3' (antisense) for E-cadherin, 5'-TTTGATGGAGGT CTCCTAACACC-3' (sense) and 5'-ACGTTTAACACGTTG GAAATGTG-3' (antisense) for N-cadherin, 5'-AGGCAA AGCAGGAGTCCACTGA-3' (sense) and 5'-ATCTGGCGT TCCAGGGACTCAT-3' (antisense) for Vimentin, 5'-TGCCCT CAAGATGCACATCCGA-3' (sense) and 5'-GGGACAGGA GAAGGGCTTCTC-3' (antisense) for Snail, 5'-TCCTCC TCTTCCTCTATACTAAC-3' (sense) and 5'-CCCACAATC ACAGGCATAG-3' (antisense) for Nanog, 5'-CTTGAATCC CGAATGGAAAGGG-3' (sense) and 5'-GTGTATATCCCA GGGTGATCCTC-3' (antisense) for Oct4, 5'-GTAACCCGT TGAACCCCATT-3' (sense) and 5'-CCATCCAATCGGTAG TAGCG-3' (antisense) for 18S, 5'-GTATTGGGCGCCTGG TCACC-3' (sense) and 5'-CGCTCCTGGAAGATGGTGA TGG-3' (antisense) for GAPDH. Each sample was examined in triplicate. All the data were analysed using the comparative threshold cycle (CT) $\left(2^{-\Delta \Delta C T}\right)$ method.

siRNA transfection and lentiviral-mediated gene overexpression. To knock down MEG3 expression, we transfected PANC-1 cells (which expressed relatively high levels of MEG3) with three different interfering siRNAs (RiboBio): siMEG3-1, 5'GGCCUUCCUGAACACCUU ADTDT3' and 3'DTDTCCGGAAGGACUUGUGGAAU5'; siMEG3-2, 5'GACGUGACAAGCAGGACAUDTDT3' and 3'DTDTCUGCACUGUUCGUCCUGUA5'; and siMEG3-3, 5'CCUCUAGCUUGGAAAUGAADTDT3' and 3'DTDTGG AGAUCGAACCUUUACUU5'. A $30 \mathrm{nM}$ mixture of three siRNAs was used to avoid off-target effect and enhance the knockdown efficiency. A negative control siRNA (NC) was also purchased from the same company. These siRNAs were transfected using Lipofectamine 2000 according to the manufacturer's protocol. 
The full-length MEG3 sequence was subcloned into a lentivirus vector designed by GeneChem (Shanghai, China). MIA PaCa-2 and T3M4 cells (which exhibited relatively low levels of MEG3) were stably transfected with lentivirus containing either Lenti-MEG3-GFP or empty vector (Lenti-GFP). Transfection efficiencies were verified using qPCR.

Cell proliferation assay. The CCK-8, colony formation and EdU incorporation assays were adopted to evaluate the effect of MEG3 on cell proliferation regulation.

For the CCK-8 assay, transfected cells were plated in 96-well plates at a density of 3,000-5,000 cells/well. At 24, 48 and $72 \mathrm{~h}$ after plating, the cells were incubated with WST-8 reagent $\left(10 \mu \mathrm{l}\right.$ per well) for $2 \mathrm{~h}$ at $37^{\circ} \mathrm{C}$ in the dark, and the absorbance at $450 \mathrm{~nm}$ was then measured in each well using a microplate reader (Bio-Rad Laboratories, Hercules, CA, USA) to determine cell viability.

For the colony formation assay, transfected cells were plated into 6-well plates at a density of 200 cells/well. After 2-3 weeks, representative colonies were imaged, and the number of colonies was carefully counted.

For the EdU incorporation assay, transfected cells were plated into 96-well plates at a density of 3,000 cells/well. At $24 \mathrm{~h}$ after plating, EdU reagent from an EdU Apollo ${ }^{\circledR} 567$ In Vitro Imaging kit (RiboBio) was added to the medium following the manufacturer's instructions and incorporated into cells in the DNA synthesis phase ( $\mathrm{S}$ phase) of the cell cycle. Ten randomly chosen visual fields were observed, and the percentage of EdU-incorporated cells was calculated. Each experiment was performed in triplicate.

Boyden chamber assay. Cell migration was measured using uncoated Boyden chambers, and cell invasion was assessed using Matrigel-coated chambers. Boyden chambers (8- $\mu \mathrm{m}$ pore size, BD Biosciences, San Jose, CA, USA) were purchased from Corning Inc. (Corning, NY, USA). For the migration assays, 15,000 transfected cells suspended in serum-free medium were placed on the non-coated membrane in the top chamber. For the invasion assay, 30,000 transduced cells suspended in serum-free medium were placed in the top chamber of inserts previously coated with a 1:7 dilution of BD Matrigel matrix. Medium supplemented with 10\% FBS was added to the lower chambers and used as a chemoattractant. After 36 to $48 \mathrm{~h}$, nonmigratory cells were removed from the upper chambers using a cotton swab. The cells on the lower surface of the insert were fixed with methanol and stained with DAPI. The cell numbers were determined by counting the penetrating cells under an inverted fluorescence microscope at x200 magnification in 5 random fields per well. Each experiment was performed in triplicate.

Sphere formation assay. As anchorage-independent growth is a common characteristic of the CSC phenotype, the sphere-forming ability of the cells was evaluated by performing a sphere-forming assay using ultralow attachment culture plates (Corning Inc.) in a serum-free floating culture system. Single cell suspensions were seeded in 6-well ultralow attachment plates at a density of 10,000 cells per well in serum-free DMEM:F12 medium (Gibco) supplemented with 20 ng/ml EGF (PeproTech,
Rocky Hill, NJ, USA), 100 ng/ml bFGF (PeproTech), 1:50 B27 supplement (Gibco), $100 \mathrm{U} / \mathrm{ml}$ penicillin and $100 \mu \mathrm{g} / \mathrm{ml}$ streptomycin. After 14 days, the formed spheres, which were typically $>75 \mathrm{~mm}$ in diameter, were counted using an inverted fluorescence microscope.

Flow cytometric analysis. PC cells that express CD24, CD44 and epithelial-specific antigen (ESA) are identified as cancer stem cells (CSCs) and only compose $0.2-0.8 \%$ of the tumour. CSCs possess self-renewal and differentiation properties and are more tumourigenic than traditional cancer cells (18). To detect CSCs, the following antibodies were used: anti-CD24-APC, anti-CD44-PE, anti-ESA-PE-Vio770, IgG1-PE, IgG1-APC and IgG1-PE-Vio770 (Miltenyi Biotec, Germany). After the transfected cells were dissociated with trypsin and washed with PBS, they were incubated with the above mentioned antibodies according to the manufacturer's instructions and measured using flow cytometry (FACSCalibur, BD Biosciences). All the data were analysed with BD FACSDiva software.

Chemosensitivity assay. A total of 3,000 transfected cells were seeded in 96-well plates. At $24 \mathrm{~h}$ after plating, different doses of gemcitabine (Eli Lilly, Indianapolis, IN, USA) were added to the plates depending on the cell type, and the cells were then cultured for another $72 \mathrm{~h}$. Cell viability was determined using a CCK-8 kit, and the absorbance at $450 \mathrm{~nm}$ was measured using a microplate reader. The cell inhibition rate was calculated using the following formula: Inhibition rate $=(1-$ absorbance of treated cells/absorbance of control cells) x 100\%. Each experiment was performed in triplicate.

Western blot analysis. Total proteins were extracted from transfected cells using RIPA lysis. The protein concentration was measured using the BCA assay (Thermo Fisher Scientific, Inc., Waltham, MA, USA), and proteins (20 $\mu \mathrm{g} /$ lane) were separated on a $10 \%$ gel using SDS-PAGE and transferred to PVDF membranes. After the membranes were then blocked in $5 \%$ fat-free milk in TBST buffer $(0.1 \%$ Tween-20) for $2 \mathrm{~h}$ at room temperature, they were incubated with primary antibodies against E-cadherin (1:2,000, Cell Signaling Technology, Danvers, MA, USA), N-cadherin (1:2,000, Cell Signaling Technology), Vimentin (1:2,000, Cell Signaling Technology), Snail (1:2,000, Cell Signaling Technology), proliferating cell nuclear antigen (PCNA) (1:500, Santa Cruz Biotechnology, Dallas, TX, USA), and $\beta$-actin (1:40,000, MBL, USA) at $4^{\circ} \mathrm{C}$ overnight followed by incubation with HRP-conjugated secondary antibodies (Jackson Laboratory, Bar Harbor, ME, USA) at $4^{\circ} \mathrm{C}$ for $1 \mathrm{~h}$. Protein bands were detected using chemiluminescence (Millipore, Billerica, MA, USA) and exposure to X-ray film.

Statistical analysis. All the data are expressed as the mean \pm standard deviation (SD). Differences between groups were determined using the Student's t-test. Kaplan-Meier analysis was applied to evaluate the prognostic significance of MEG3 expression regarding patient survival. The data from the clinicopathological characteristics analysis was calculated using the Fisher exact probability test. All statistical analyses were performed using SPSS 13.0 software (SPSS Inc. Chicago, 
Table I. Relationship between MEG3 expression level and the clinicopathological characteristics of the PC cases.

\begin{tabular}{|c|c|c|c|c|}
\hline \multirow[b]{2}{*}{ Characteristics } & \multirow[b]{2}{*}{ Number of cases $(n=25)$} & \multicolumn{2}{|c|}{ MEG3 expression level } & \multirow[b]{2}{*}{ P-value } \\
\hline & & Low $(n=16)(\%)$ & $\operatorname{High}(n=9)(\%)$ & \\
\hline Age (years) & & & & 0.243 \\
\hline$<60$ & 12 & $8(50)$ & $4(44.44)$ & \\
\hline$\geq 60$ & 13 & $8(50)$ & $5(55.56)$ & \\
\hline Sex & & & & 0.560 \\
\hline Male & 11 & $7(43.75)$ & $4(44.44)$ & \\
\hline Female & 14 & $9(56.25)$ & $5(55.56)$ & \\
\hline Tumour size & & & & 0.407 \\
\hline$<4 \mathrm{~cm}$ & 16 & $11(68.75)$ & $5(55.56)$ & \\
\hline$\geq 4 \mathrm{~cm}$ & 9 & $5(31.25)$ & $4(44.44)$ & \\
\hline Location & & & & 0.626 \\
\hline Head of pancreas & 17 & $11(68.75)$ & $6(66.67)$ & \\
\hline Body and tail of pancreas & 8 & $5(31.25)$ & $3(33.33)$ & \\
\hline Histological grade & & & & 0.174 \\
\hline Well and moderate & 7 & $6(37.5)$ & $1(11.11)$ & \\
\hline Poor & 18 & $10(62.5)$ & $8(88.89)$ & \\
\hline Primary tumour & & & & 0.542 \\
\hline $\mathrm{T} 1, \mathrm{~T} 2$ & 4 & $3(18.75)$ & $1(11.11)$ & \\
\hline $\mathrm{T} 3, \mathrm{~T} 4$ & 21 & $13(81.25)$ & $8(88.89)$ & \\
\hline Lymph node & & & & 0.626 \\
\hline No & 17 & $11(68.75)$ & $6(66.67)$ & \\
\hline $\mathrm{N} 1$ & 8 & $5(31.25)$ & $3(33.33)$ & \\
\hline Venous invasion & & & & 0.593 \\
\hline Absent & 16 & $10(37.5)$ & $6(66.67)$ & \\
\hline Present & 9 & $6(62.5)$ & $3(33.33)$ & \\
\hline Neural invasion & & & & 0.144 \\
\hline Absent & 4 & $4(25)$ & $0(0)$ & \\
\hline Present & 21 & $12(75)$ & $9(100)$ & \\
\hline CA19-9 & & & & 0.230 \\
\hline$<37 \mathrm{U} / \mathrm{ml}$ & 5 & $2(12.5)$ & $3(33.33)$ & \\
\hline$\geq 37 \mathrm{U} / \mathrm{ml}$ & 20 & $14(87.5)$ & $6(66.67)$ & \\
\hline CEA & & & & 0.593 \\
\hline$<5 \mathrm{ng} / \mathrm{ml}$ & 16 & $10(62.5)$ & $6(66.67)$ & \\
\hline$\geq 5 \mathrm{ng} / \mathrm{ml}$ & 9 & $6(37.5)$ & $3(33.33)$ & \\
\hline CA-242 & & & & 0.098 \\
\hline$<5 \mathrm{ng} / \mathrm{ml}$ & 11 & $5(31.25)$ & $6(66.67)$ & \\
\hline$\geq 5 \mathrm{ng} / \mathrm{ml}$ & 14 & $11(68.75)$ & $3(33.33)$ & \\
\hline
\end{tabular}

PC, pancreatic cancer; CA19-9, cancer antigen 19-9; CEA, carcinoembryonic antigen; CA-242, cancer antigen 242.

IL, USA). A P-value $<0.05$ was considered to indicate a statistically significant difference.

\section{Results}

Decreased expression level of MEG3 is detected in both PC tissues and cell lines and is correlated with worse prognosis. We isolated pure malignant pancreatic ducts from frozen PC sections using the LCM technique. Total RNA was successfully extracted from 25 microdissected tissues, and
MEG3 expression was examined using qPCR. Compared to MEG3 expression in the adjacent normal tissues (ANT), MEG3 expression was significantly decreased in $64 \%$ of the PC tumours (16/25) (Fig. 1A). Absence of expression of MEG3 was observed in most of the PC cell lines when compared with that in the hTERT-HPNE cells, except for PANC-1 cells (Fig. 1B). Although the statistical analysis failed to identify any significant correlation between the clinicopathological characteristics and the MEG3 expression level (Table I), Kaplan-Meier analysis revealed that patients with 

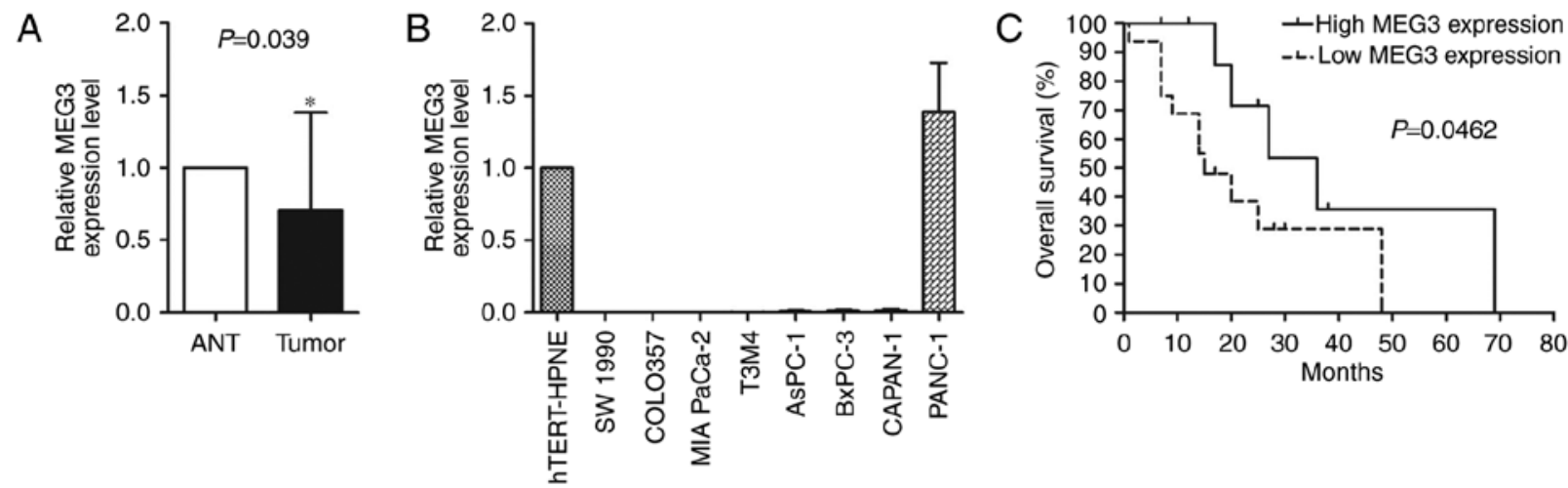

Figure 1. Decreased MEG3 expression is shown in PC samples and cell lines, which was associated with a worse prognosis in PC patients. The expression of MEG3 in microdissected PC tissues (A) and PC cell lines (B) was examined using qPCR. (C) The Kaplan-Meier method was used to analyse overall survival and to produce survival curves. The results are presented as the mean $\pm \mathrm{SD}, \mathrm{n}=3,{ }^{*} \mathrm{P}<0.05$. PC, pancreatic cancer; ANT, adjacent normal tissue.

relatively elevated MEG3 expression exhibited a longer overall survival time $(\mathrm{P}<0.05)$ (Fig. 1C).

Effect of MEG3 on cell proliferation, migration and invasion in PC cells. To investigate the functional role of MEG3 in the tumour progression of $\mathrm{PC}$, we established three transfected cell lines in which MEG3 was differentially expressed. PANC-1 cells, which naturally express relatively high levels of MEG3, were transfected with siRNAs to knock down MEG3 expression; MIA PaCa-2 and T3M4 cells were stably transfected with a lentivirus vector that overexpresses MEG3. qPCR confirmed that transfection of the siMEG3 mixture reduced the MEG3 mRNA levels by $80 \%$, whereas cells with the Lenti-MEG3-GFP vector exhibited stably increased MEG3 mRNA levels by two orders of magnitude compared to the levels in the control group (Fig. 2A).

The CCK-8 assay suggested that knockdown of MEG3 expression in PANC-1 cells promoted cell growth, especially at $72 \mathrm{~h}$ after transfection. Conversely, ectopic expression of MEG3 in MIA PaCa-2 and T3M4 cells significantly decreased cell viability and growth (Fig. 2C). The colony formation ability of plated transfected cells was also affected by the levels of MEG3 expression. The number of colonies formed by PANC-1 cells (which were transfected with siMEG3s) was increased; however, the number and size of the colonies formed by MIA PaCa-2 and T3M4 cells transfected with Lenti-MEG3-GFP decreased by nearly 50\% (Fig. 2D). The EdU incorporation assay showed that the knockdown of MEG3 in PANC-1 cells upregulated the percentage of cells in the $\mathrm{S}$ phase by $10 \%$, whereas overexpression of this lncRNA downregulated the percentage of MIA PaCa-2 and T3M4 cells in $\mathrm{S}$ phase (Fig. 2B).

The influence of MEG3 on the migratory and invasive abilities of PC cells was examined using the Boyden chamber assay. MEG3 knockdown in PANC-1 cells significantly enhanced cell migration and invasion to a large extent, especially the invasive ability. However, ectopic overexpression of MEG3 in MIA PaCa-2 and T3M4 elicited an opposing effect (Fig. 2E and F).

Effect of MEG3 on epithelial-mesenchymal transition in $P C$ cells. To elucidate the mechanisms by which MEG3 overexpression suppresses cell migration and invasion, we examined the mRNA (Fig. 3A) and protein levels (Fig. 3B) of several key genes involved in EMT. The qPCR and western blot results consistently showed that both the transcription and translation of genes involved in EMT were affected when MEG3 expression was altered. Knockdown of MEG3 in PANC-1 cells decreased E-cadherin expression while inducing N-cadherin, Vimentin and Snail expression. Inversely, inhibition of EMT process was observed in MIA PaCa-2 and T3M4 cells with ectopic overexpression of MEG3. Meanwhile, we examined the protein levels of PCNA, which reflects the proliferative state of the cells, and found that PCNA expression was suppressed in cells with MEG3 overexpression.

Effects of MEG3 on the sphere-forming ability, the CSC properties and the chemosensitivity in PC cells. As the MIA PaCa-2 cell line showed no ability to form stem cell spheres, the sphere-forming assay was performed using transfected PANC-1 and T3M4 cells. PANC-1 cells with MEG3 knockdown exhibited an increased number and size of spheres when compared to the control cells, whereas ectopic expression of MEG3 in T3M4 cells decreased their sphere-forming ability (Fig. 4A). CSCs expressing CD24, CD44 and ESA were detected in the three transfected cell lines using flow cytometry, and the results were concordant with those of the sphere-forming assay (Fig. 4B). The percentage of $\mathrm{CD} 24^{+} \mathrm{CD} 44^{+} \mathrm{ESA}^{+} \mathrm{CSC}$ in PANC-1 cells was significantly increased from $1.39 \pm 0.64 \%$ to $2.64 \pm 0.77 \%$, when MEG3 was downregulated $(\mathrm{P}=0.049)$. And the opposite effects were shown in both MIA PaCa-2 and T3M4 cells overexpressing MEG3 (Fig. 4C). Nanog and Oct4 have been identified as mature markers of CSCs, and the qPCR results showed that downregulation of MEG3 in PANC-1 cells slightly increased the mRNA expression of Nanog and Oct4, whereas upregulation of MEG3 significantly decreased these genes in MIA PaCa-2 and T3M4 cells (Fig. 4D). Gemcitabine is a first-line chemotherapeutic agent used in PC. As shown in Fig. 4E, upregulation of MEG3 enhanced the chemosensitivity of MIA $\mathrm{PaCa}-2$ and T3M4 cells to gemcitabine, whereas downregulation of MEG3 increased the chemoresistance of PANC-1 cells. In the transduced cell lines, the $\mathrm{IC}_{50}$ of gemcitabine 
A

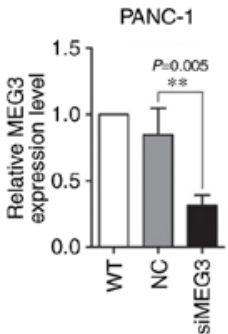

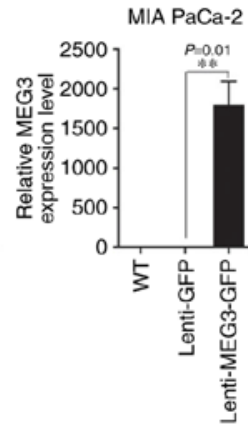

C

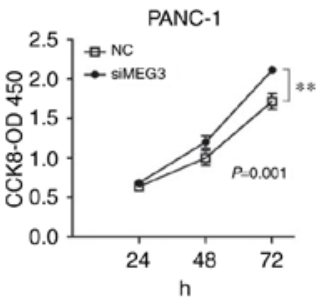

T3M4

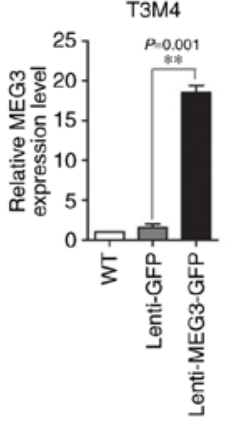

B

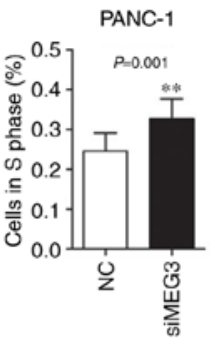

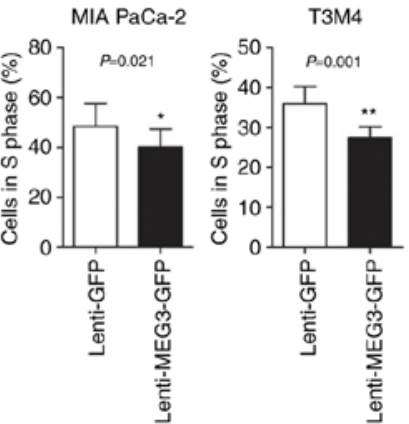

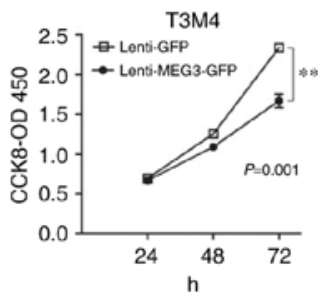

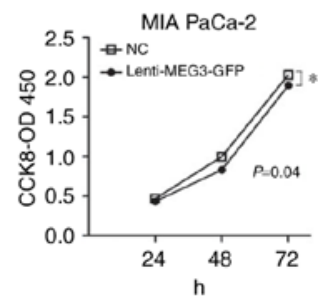
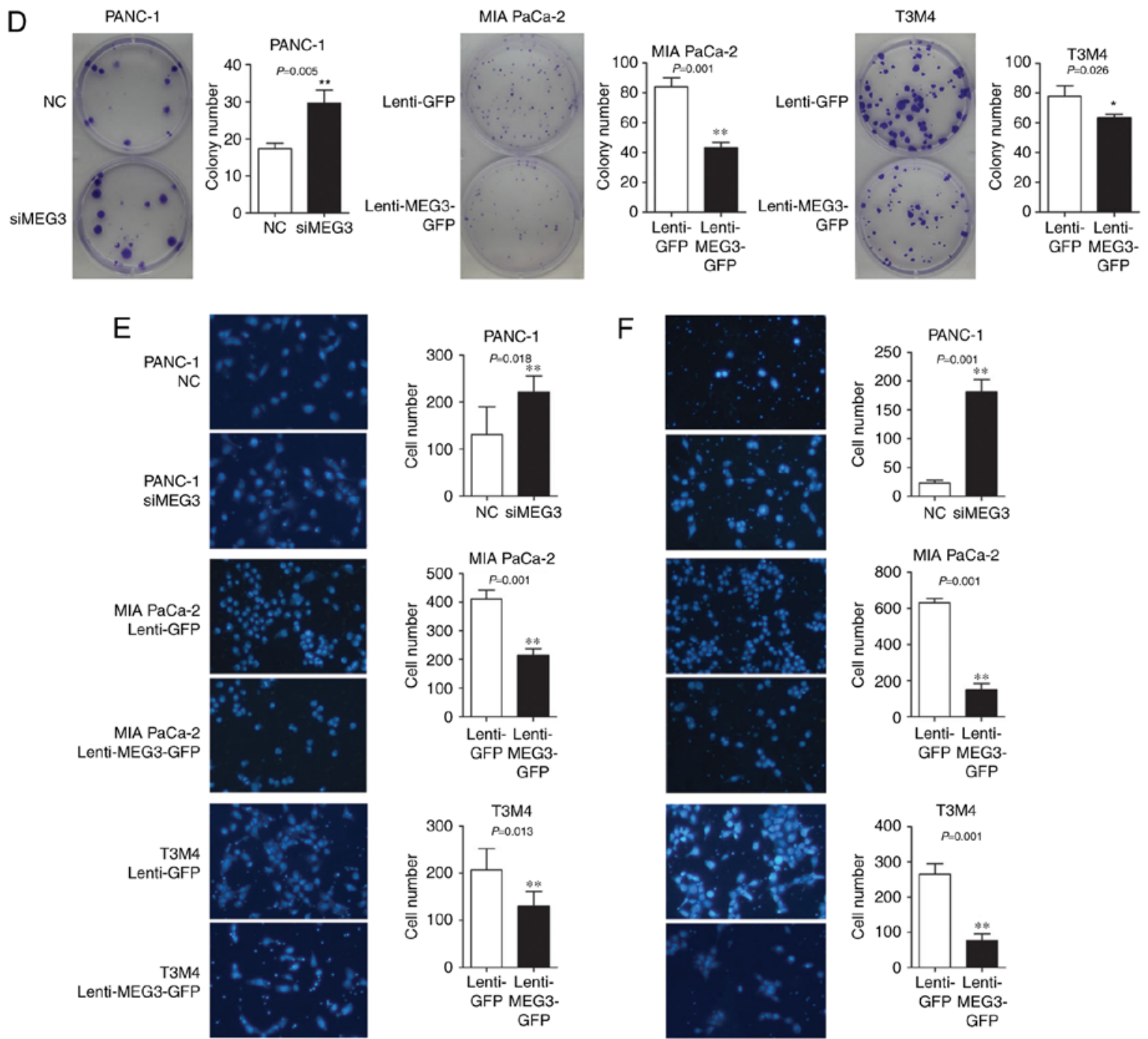

$\mathrm{F}$
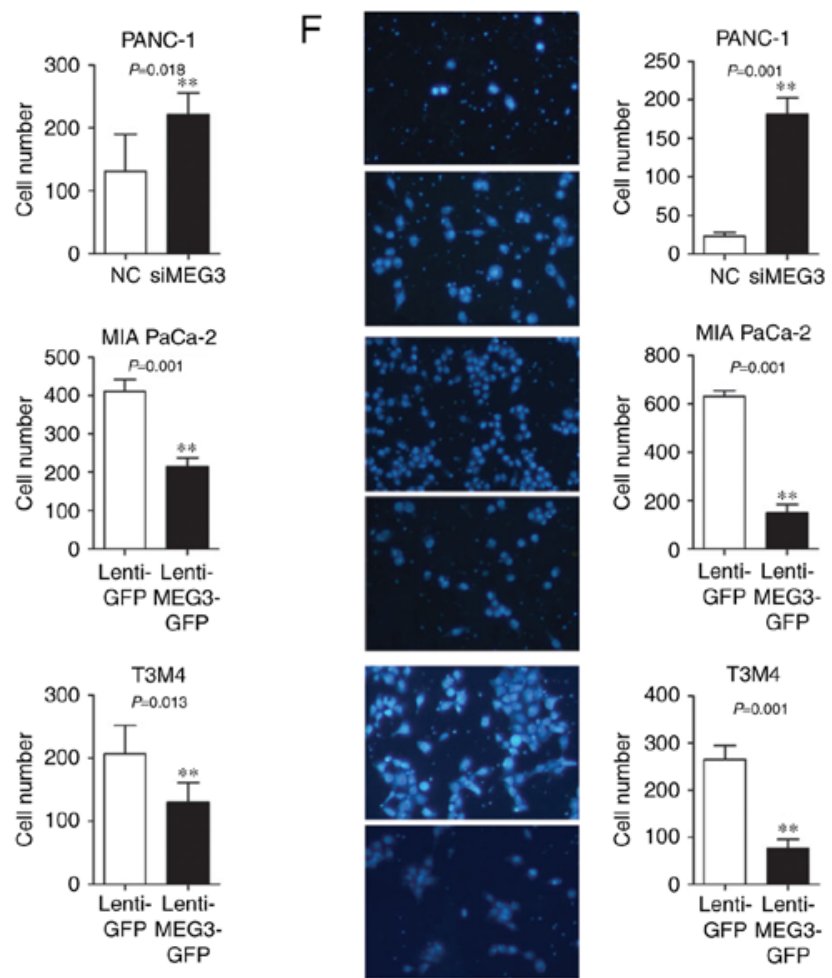

Figure 2. MEG3 regulates cell proliferation, migration and invasion in PC in vitro. (A) PANC-1 cells were transfected with either a siMEG3 mixture or siNC, and MIA PaCa-2 and T3M4 cells were transfected with either Lenti-MEG3-GFP or Lenti-GFP. The transfection efficiency was verified using qPCR. (B) The EdU incorporation assay indicated the percentage of cells in the S phase. Cells that incorporated EdU were detected using fluorescence microscopy and counted. (C) Cell viability was assessed using the CCK-8 assay. The cell proliferation curves were drawn based on the absorbance at $450 \mathrm{~nm}$ measured every $24 \mathrm{~h}$ after transfection. (D) A total of 200 transfected cells were plated in 6-well plates and cultured for 14 days, and the number of macroscopic colonies was counted. (E) The transfected cells were seeded in the top chamber of uncoated Boyden inserts to evaluate the migratory ability. (F) The transfected cells were seeded in the top chamber of Matrigel-coated inserts to evaluate the invasive ability. The bar graph represents the number of migratory or invasive cells per 5 random fields. The results are expressed as the mean $\pm \mathrm{SD}, \mathrm{n}=3,{ }^{*} \mathrm{P}<0.05,{ }^{* * *} \mathrm{P}<0.01$. $\mathrm{PC}$, pancreatic cancer. 
A

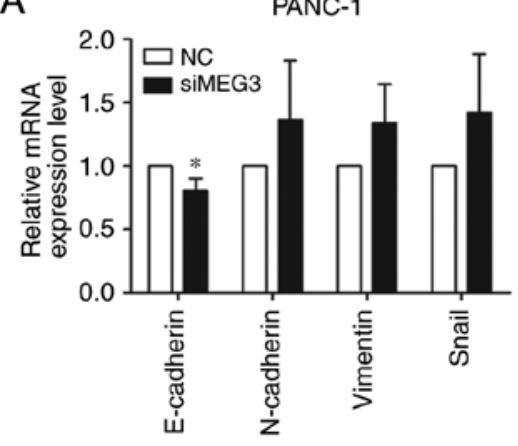

B

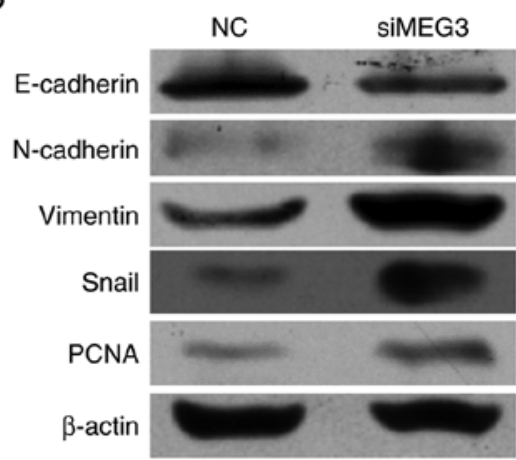

MIA PaCa-2

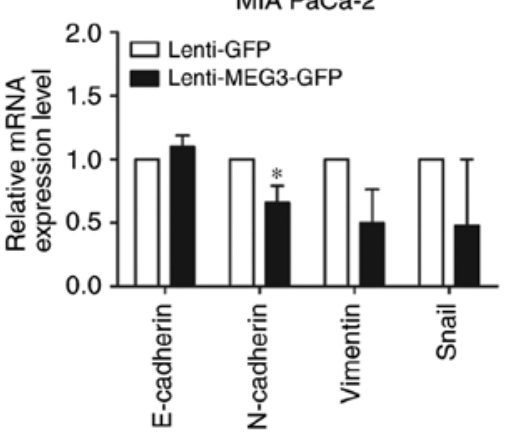

MIA PaCa-2

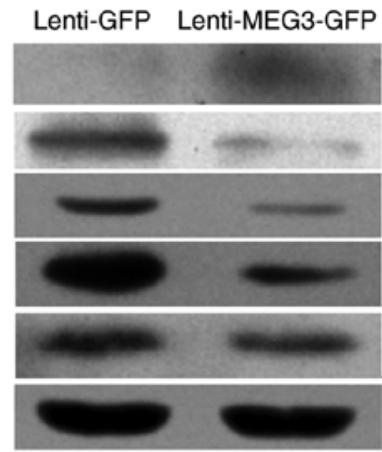

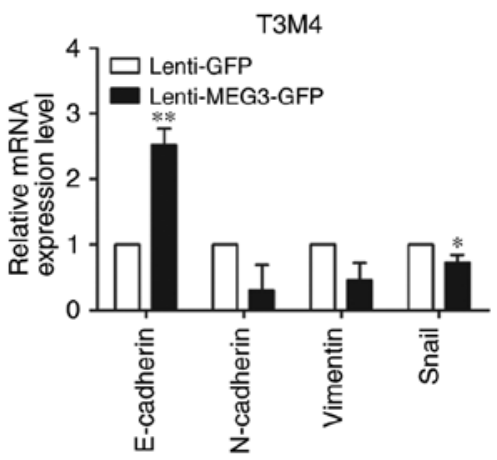

T3M4

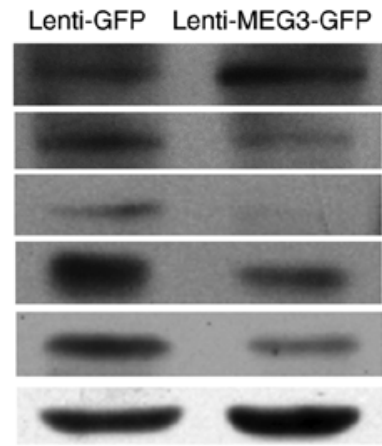

Figure 3. MEG3 regulates EMT at the transcriptional and translational levels in PC cells. The mRNA levels (A) and protein levels (B) of E-cadherin, $\mathrm{N}$-cadherin, Vimentin, Snail and PCNA were determined using $\mathrm{qPCR}$ and western blot analysis. The results are expressed as the mean $\pm \mathrm{SD}$, $\mathrm{n}=3$, ${ }^{*} \mathrm{P}<0.05$, ${ }^{* *} \mathrm{P}<0.01$. EMT, epithelial-mesenchymal transition; $\mathrm{PC}$, pancreatic cancer.

decreased from $1,065.33 \pm 170.85 \mathrm{ng} / \mathrm{ml}$ to $307.66 \pm 39.37 \mathrm{ng} / \mathrm{ml}$ in MIA PaCa-2 cells and from $1,946.05 \pm 217.42 \mathrm{ng} / \mathrm{ml}$ to $660.57 \pm 57.38 \mathrm{ng} / \mathrm{ml}$ in T3M4 cells, but increased from $95.87 \pm 7.20 \mu \mathrm{g} / \mathrm{ml}$ to $184.19 \pm 8.13 \mu \mathrm{g} / \mathrm{ml}$ in PANC-1 cells $(\mathrm{P}<0.05)$ (Fig. 4F).

\section{Discussion}

Accumulating evidence has identified MEG3 as a tumour suppressor in various types of cancers. However, the relationship between MEG3 and human PC remains poorly understood. In this study, we focused on identifying the functional role of MEG3 in PC and provided comprehensive data that the loss of MEG3 expression in PC could promote tumourigenesis with respect to cell proliferation, EMT, CSC properties and chemoresistance.

Consistent with results in other malignancies, the significantly decreased level of MEG3 expression was observed in microdissected PC samples and cancer cell lines compared to normal control tissues and cells. Although we failed to find a significant correlation between MEG3 expression and the clinicopathological features in the PC cases in this study (likely because of the small sample size), our data suggested that the MEG3 expression level was significantly associated with the overall survival rate in PC patients. Compared with those with a relatively high level of MEG3, the patients with a low level of MEG3 seemed to be correlated with a worse outcome. This means that MEG3 may act as a tumour suppressor and predict prognosis in human $\mathrm{PC}$, and the specific mechanisms of MEG3 in PC cells merit further investigation.
The tumour suppressor property of MEG3 has been demonstrated in many malignancies, and the corresponding mechanisms mainly involve the modulation of cell proliferation. In addition to the high proliferation activity, early distant metastasis and chemoresistance also contribute to the poor prognosis of PC patients. However, less is known concerning the effects of MEG3 on tumour metastasis and chemoresistance. In the present study, we investigated the biological functions of MEG3 on cell proliferation, cell migration and invasion, EMT, CSC properties and chemoresistance in PC cells. In addition to verifying the previous reports that MEG3 has a significantly negative correlation with the proliferation modulation, our results also indicated that decreased expression of MEG3 could promote PC cell migration and invasion, as well as chemoresistance by regulating the EMT process and CSC properties.

The EMT process has been considered as a hallmark of the metastasis cascade of malignancies. During the process of EMT, immobile epithelial cells are transformed into motile mesenchymal cells and then gain the ability to invade through the stroma. Meanwhile, cancer cells undergoing EMT often acquire stemness characteristics, which exhibit the properties of dissemination and self-renewal required for the initiation of a secondary tumour (19). The CSCs, which account for a very small proportion of the correspond cancer cells, are naturally resistant to most chemotherapeutic agents. Moreover, numerous studies suggest that chemoresistant cancer cells exhibit a mesenchymal phenotype $(20,21)$. Izumiya et al reported that chemoresistant PC cells present CSC traits, as these cells initiate sphere formation, express stem cell markers 

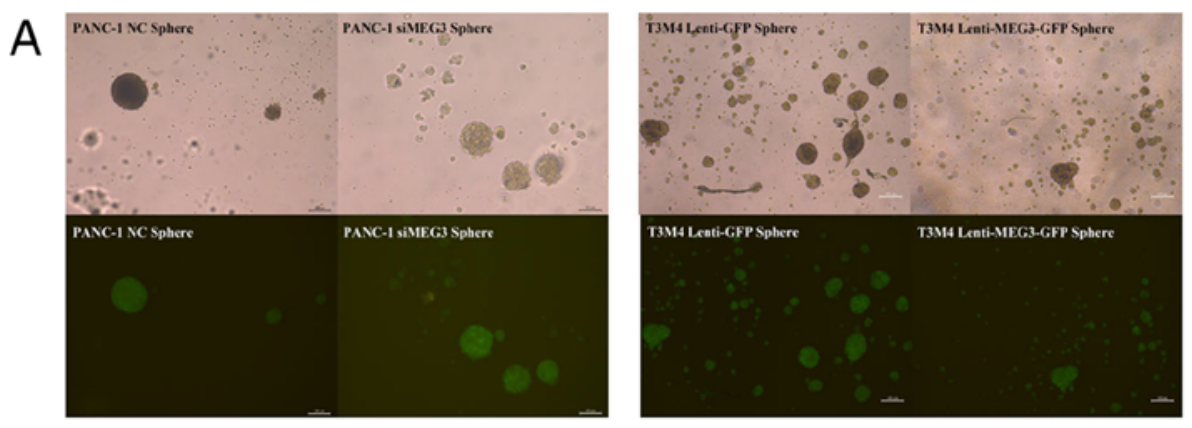

B
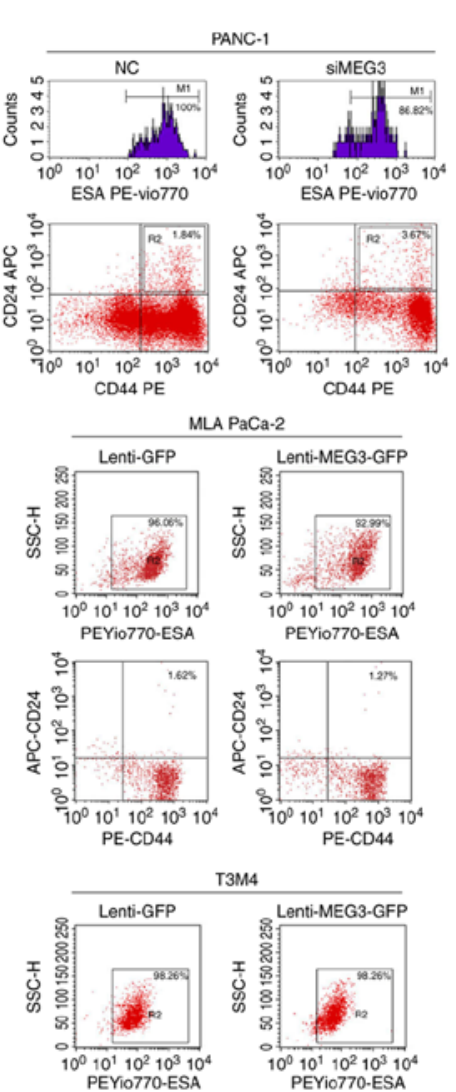

Тзм4
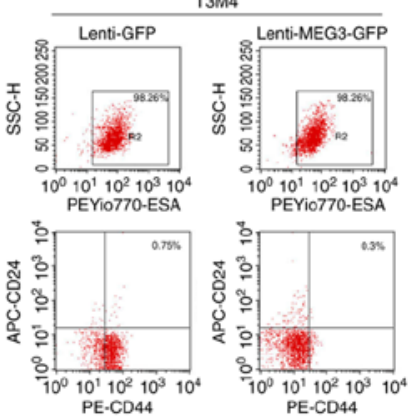

C
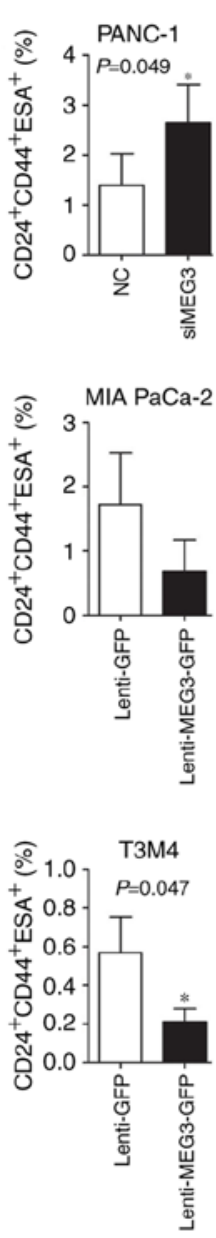

E
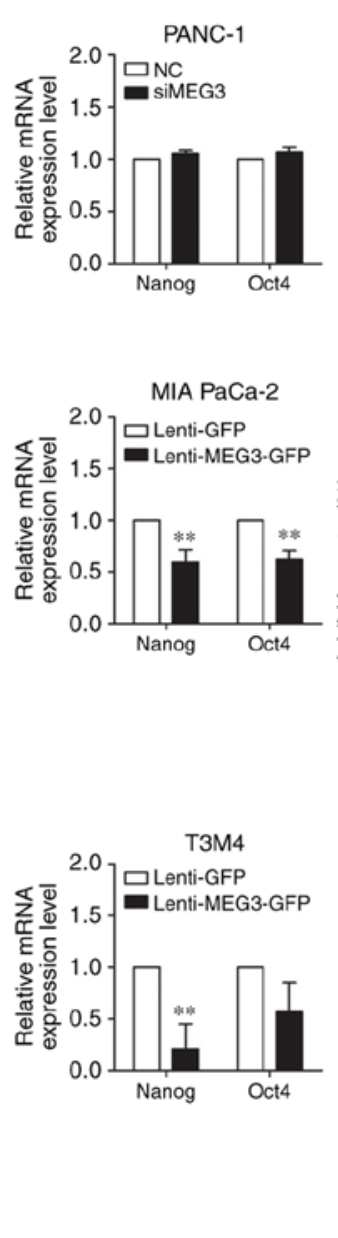
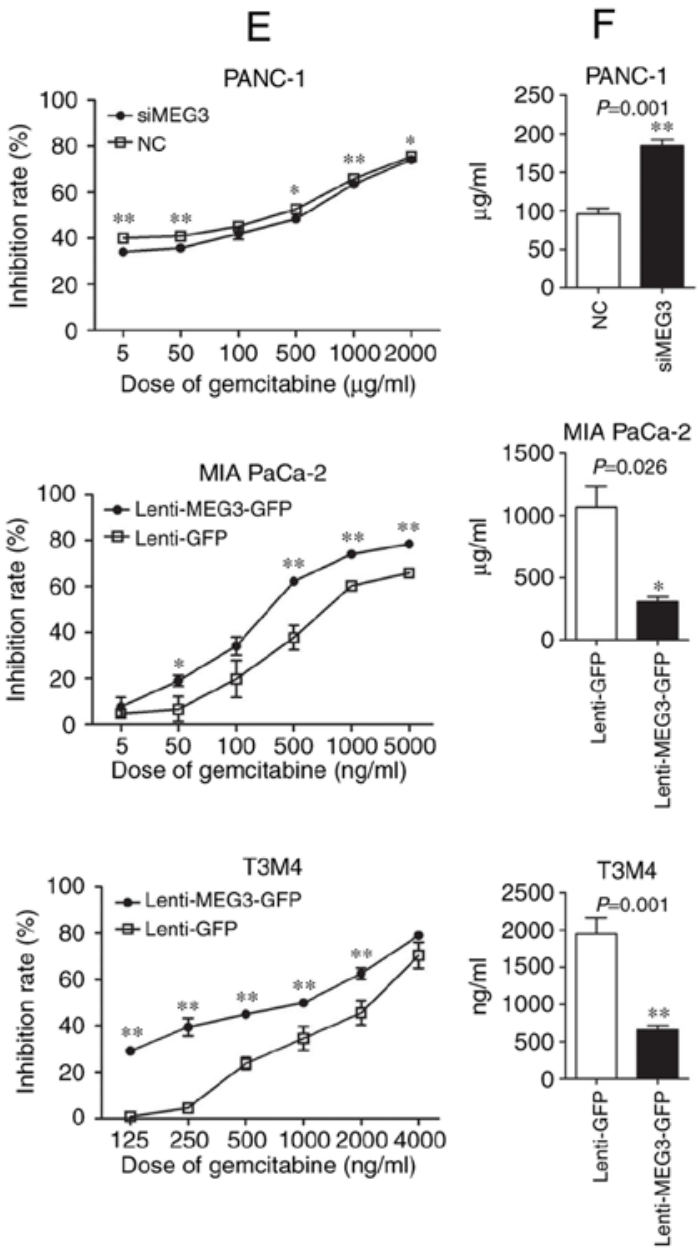

Figure 4. MEG3 regulates the sphere-forming ability, CSC properties and chemosensitivity in PC cells. (A) A serum-free floating culture system was adopted to examine the sphere-forming ability. Images were taken of cells on an inverted fluorescence microscope after the cells were cultured for 14 days in ultralow attachment plates, and the number of formed spheres $>75 \mathrm{~mm}$ was counted. (B) CD24+CD44+ESA ${ }^{+}$cells were detected using flow cytometry after transfection. (C) The percentage of $\mathrm{CD} 44^{+} \mathrm{CD} 24^{+} \mathrm{ESA}^{+}$cells among the transfected cells compared with cells in the control group. (D) Nanog and Oct4 expression levels were measured using qPCR. (E) Inhibition curves of transfected cells at different doses of gemcitabine. (F) The $\mathrm{IC}_{50}$ of gemcitabine in transfected cells compared with cells in the control group. The results are expressed as the mean $\pm \mathrm{SD}, \mathrm{n}=3,{ }^{*} \mathrm{P}<0.05,{ }^{* *} \mathrm{P}<0.01$.

and respond to EMT stimulation (22). Taken together, all these data indicate that EMT, CSC properties and chemoresistance are interrelated and inextricably linked to tumourigenesis. Our results revealed that the loss of MEG3 expression in PC cells might be involved in the initiation of chemoresistance, EMT and CSC properties, and is associated with a worse patient prognosis.
The molecular mechanisms of MEG3 in regards to cell proliferation, cell migration and invasion, EMT, CSC properties and chemoresistance in PC cells warrant further investigation. In this preliminary study, we found that differential expression of MEG3 significantly altered the mRNA and protein levels of Snail, a key transcription factor that has been reported to be associated with EMT and stemness 
maintenance. Considering that MEG3 is a ncRNA which functions as a regulator of epigenetic modifications, transcriptional or post-transcriptional regulation, we could speculate that Snail might be a potential downstream target of MEG3 that regulates PC progression. Additional studies are needed to elucidate how Snail is regulated by MEG3 in human PC.

In conclusion, our findings suggest that MEG3 is expressed at low levels in human PC and is associated with patient prognosis. The in vitro experiments illustrate that MEG3 could function as a tumour suppressor by modulating cell proliferation, cell migration and invasion, EMT, CSC properties and chemosensitivity. Therefore, MEG3 has great potential to serve as a prognostic predictor and therapeutic target for individuals with PC.

\section{Acknowledgements}

This research was supported by grants from the National Natural Science Foundation of China (nos. 81372605, 81572339, and 81672353), the National High Technology Research and Development Program of China (SS2014AA020405) and the Scientific Research and Development Program of Beijing Railway Corporation of China (no. J2017Z605). We thank Professor Zebin Mao at the Department of Biochemistry and Molecular Biology in Health Science Center, Peking University for the assistance and technical support.

\section{References}

1. Siegel RL, Miller KD and Jemal A: Cancer Statistics, 2017. CA Cancer J Clin 67: 7-30, 2017.

2. Ferlay J, Partensky C and Bray F: More deaths from pancreatic cancer than breast cancer in the EU by 2017. Acta oncol 55: $1158-1160,2016$

3. Miyoshi N, Wagatsuma H, Wakana S, Shiroishi T, Nomura M, Aisaka K, Kohda T, Surani MA, Kaneko-Ishino T and Ishino F: Identification of an imprinted gene, Meg3/Gtl2 and its human homologue MEG3, first mapped on mouse distal chromosome 12 and human chromosome 14q. Genes cells 5: 211-220, 2000

4. Zhang X, Zhou Y, Mehta KR, Danila DC, Scolavino S, Johnson SR and Klibanski A: A pituitary-derived MEG3 isoform functions as a growth suppressor in tumor cells. J Clin Endocrinol Metab 88: 5119-5126, 2003.

5. Cheng R, Lo K, Huang D and Tsao S: Loss of heterozygosity on chromosome 14 in primary nasopharyngeal carcinoma. Int J Oncol 10: 1047-1050, 1997.

6. Liu J, Wan L, Lu K, Sun M, Pan X, Zhang P, Lu B, Liu G and Wang Z: The long noncoding RNA MEG3 contributes to cisplatin resistance of human lung adenocarcinoma. PLoS One 10: e114586, 2015.

7. Bando T, Kato Y, Ihara Y, Yamagishi F, Tsukada K and Isobe M: Loss of heterozygosity of 14q32 in colorectal carcinoma. Cancer Genet Cytogenet 111: 161-165, 1999.
8. Yin DD, Liu ZJ, Zhang E, Kong R, Zhang ZH and Guo RH: Decreased expression of long noncoding RNA MEG3 affects cell proliferation and predicts a poor prognosis in patients with colorectal cancer. Tumour Biol 36: 4851-4859, 2015.

9. Braconi C, Kogure T, Valeri N, Huang N, Nuovo G, Costinean S, Negrini M, Miotto E, Croce CM and Patel T: MicroRNA-29 can regulate expression of the long non-coding RNA gene MEG3 in hepatocellular cancer 30: 4750-4766, 2011.

10. Greife A, Knievel J, Ribarska T, Niegisch G and Schulz WA: Concomitant downregulation of the imprinted genes DLK1 and MEG3 at 14q32.2 by epigenetic mechanisms in urothelial carcinoma. Clin Epigenetics 6: 29, 2014.

11. Tian ZZ, Guo XJ, Zhao YM and Fang Y: Decreased expression of long non-coding RNA MEG3 acts as a potential predictor biomarker in progression and poor prognosis of osteosarcoma. Int J Clin Exp Pathol 8: 15138-15142, 2015.

12. Itoyama T, Chaganti RS, Yamada Y, Tsukasaki K, Atogami S, Nakamura H, Tomonaga M, Ohshima K, Kikuchi $\mathbf{M}$ and Sadamori N: Cytogenetic analysis and clinical significance in adult T-cell leukemia/lymphoma: A study of 50 cases from the human T-cell leukemia virus type-1 endemic area, Nagasaki. Blood 97: 3612-3620, 2001.

13. Huarte M, Guttman M, Feldser D, Garber M, Koziol MJ, Kenzelmann-Broz D, Khalil AM, Zuk O, Amit I, Rabani M, et al: A large intergenic noncoding RNA induced by p53 mediates global gene repression in the p53 response. Cell 142: 409-419, 2010.

14. Zhao J, Zhang X, Zhou Y, Ansell PJ and Klibanski A: Cyclic AMP stimulates MEG3 gene expression in cells through a cAMP-response element (CRE) in the MEG3 proximal promoter region. Int J Biochem Cell Biol 38: 1808-1820, 2006.

15. Gordon FE, Nutt CL, Cheunsuchon P, Nakayama $Y$, Provencher KA, Rice KA, Zhou Y, Zhang X and Klibanski A: Increased expression of angiogenic genes in the brains of mouse meg3-null embryos. Endocrinology 151: 2443-2452, 2010.

16. Gao Y and Lu X: Decreased expression of MEG3 contributes to retinoblastoma progression and affects retinoblastoma cell growth by regulating the activity of Wnt/ $\beta$-catenin pathway. Tumour Biol 37: 1461-1469, 2016.

17. Chang L, Wang G, Jia T, Zhang L, Li Y, Han Y, Zhang K, Lin G, Zhang R, Li J and Wang L: Armored long non-coding RNA MEG3 targeting EGFR based on recombinant MS2 bacteriophage virus-like particles against hepatocellular carcinoma. Oncotarget 7: 23988-24004, 2016

18. Li C, Heidt DG, Dalerba P, Burant CF, Zhang L, Adsay V, Wicha M, Clarke MF and Simeone DM: Identification of pancreatic cancer stem cells. Cancer Res 67: 1030-1037, 2007.

19. Hollier BG,Evans K and ManiSA: The epithelial-to-mesenchymal transition and cancer stem cells: A coalition against cancer therapies. J Mammary Gland Biol Neoplasia 14: 29-43, 2009.

20. Shah AN,Summy JM,Zhang J,Park SI,Parikh NU and Gallick GE: Development and characterization of gemcitabine-resistant pancreatic tumor cells. Ann Surg Oncol 14: 3629-3637, 2007.

21. Wang Z, Li Y, Kong D, Banerjee S, Ahmad A, Azmi AS, Ali S, Abbruzzese JL, Gallick GE and Sarkar FH: Acquisition of epithelial-mesenchymaltransition phenotype of gemcitabine-resistant pancreatic cancer cells is linked with activation of the notch signaling pathway. Cancer Res 69: 2400-2407, 2009.

22. Izumiya M, Kabashima A, Higuchi H, Igarashi $T$, Sakai G, Iizuka H, Nakamura S, Adachi M, Hamamoto Y, Funakoshi S, et al: Chemoresistance is associated with cancer stem cell-like properties and epithelial-to-mesenchymal transition in pancreatic cancer cells. Anticancer Res 32: 3847-3853, 2012. 